\title{
Do INI1 and E-cadherin Expression Loss Have Any Significance in Endometrial Carcinomas?
}

\author{
(1) Sevil Karabağ
}

Tekirdağ Namık Kemal University Faculty of Medicine, Department of Pathology, Tekirdağ, Turkey

Cite this article as: Karabağ S. Do INI1 and E-cadherin Expression Loss Have Any Significance in Endometrial Carcinomas?.

J Acad Res Med 2021;11(1):32-37

\begin{abstract}
Objective: Endometrial carcinoma (EC) is a highly heterogeneous malignancy in terms of morphology, clinical course, response to treatment and prognosis. This study aimed to investigate the loss of INI1 (SWI/SNF family member) and E-cadherin expression in type 1 and 2 ECs to elucidate the mechanisms that may elucidate on the differences in pathogenesis and prognosis between low- and high-grade histological types.

Methods: Immunohistochemistry (IHC) was applied for INI1 and E-cadherin in 72 patients who underwent hysterectomy for EC. Loss of INI1 and E-cadherin expression was compared between subjects with low- and high-grade EC.

Results: A total of 63 patients had type 1 (endometrioid) and nine had type 2 (non-endometrioid) tumours. IHC staining revealed loss of INI1 expression in eight cases. While three of these cases were serous carcinoma, three were endometrioid carcinoma with villoglandular pattern and two were grade 3 endometrioid carcinoma. A significant difference was found in the loss of INI1 expression between low-grade (G1-G2) and highgrade ( $\mathrm{G} 3$ endometrioid carcinoma and non-endometrioid carcinoma) tumours $(p=0.004$ ). Loss of expression was observed only in one case of dedifferentiated carcinoma in IHC staining performed for E-cadherin in 72 cases.

Conclusion: A significantly greater loss of INI1 expression was observed in high-grade compared with low-grade endometrial carcinoma. This finding confirms that INI1 loss is a poor prognostic factor in these tumours as in other tumours reported in the literature and sheds light on the different pathogeneses seen in high-grade EC. To the best of our knowledge, this is the first systematic study to investigate INI 1 loss in different types of endometrial carcinoma. Our results support the notion that SWI/SNF chromatin remodelling complex plays a role in the pathogenesis of high-grade $\mathrm{EC}$ and type 1 endometrioid carcinoma with villoglandular pattern.
\end{abstract}

Keywords: Endometrial carcinoma, INI1, E-cadherin

\section{INTRODUCTION}

Endometrial carcinoma (EC) is the most common malignant tumour of the female genital system, accounting for approximately $4 \%$ of all cancers in women with an incidence of 10-20/100,000 individuals $(1,2)$. EC is divided into two main types based on pathogenesis, namely, type 1 and type 2. Type 1 endometrioid carcinomas are oestrogen-dependent low-grade neoplasms with precursor lesions (endometrial hyperplasia/endometrioid intraepithelial neoplasia) that occur in perimenopausal patients.

ORCID ID of the author: S.K. 0000-0002-8855-3798.

Corresponding Author/Sorumlu Yazar: Sevil Karabağ,

E-mail: eesevil-krbg@hotmail.com
Received Date/Geliş Tarihi: 19.11.2020 Accepted Date/Kabul Tarihi: 18.01.2021

(C) Copyright 2021 by University of Health Sciences Turkey, Gaziosmanpaşa Training and Research Hospital. Available on-line at www.jarem.org 
Type 2 non-endometrioid (mostly serous or clear-cell) carcinomas are high-grade tumours that occur in postmenopausal older patients in the absence of hyperestrogenism (3). Majority of endometrioid ECs are low-grade tumours (grades 1 and 2) that are associated with a good prognosis when limited to the uterus. Grade 3 endometrioid carcinoma is an aggressive tumour with increased lymph node metastasis. Endometrioid carcinoma grade 3 and serous carcinoma are considered high-grade tumours. Serous carcinoma and grade 3 endometrioid carcinoma constitute $39 \%$ and $27 \%$ of cancer-related deaths, respectively (4).

Although useful, this traditional classification is different from the histological classification. The increasing information about morphological heterogeneity, clinical symptoms, prognosis, response to treatment and mortality of EC has revealed the limitations of the traditional classification (3). It may be challenging to position certain EC subtypes in one of these two groups. On the contrary, molecular studies have appeared promising to obtain important information about prognosis and establish novel treatment modalities $(2,5)$.

Since serous and endometrioid carcinomas substantially differ in terms of clinical behaviour, it is imperative to distinguish serous carcinoma with papillary morphology from endometrioid carcinoma with villoglandular pattern. Villoglandular endometrioid carcinomas are assumed to behave similar to non-villoglandular endometrioid carcinomas. However, few studies have focused on the villoglandular variant of endometrioid carcinomas (6). While some studies have shown that these tumours exhibit comparable behaviour to non-villoglandular serous carcinomas with vascular invasion, potential lymph node metastasis and a more aggressive course, (7) others have suggested that they behave similar to oestrogen-dependent type 1 endometrioid carcinomas, which are not associated with poor prognosis (6).

The SWI/SNF complex is thought to be involved as a tumour suppressor gene owing to its role in chromatin remodelling and transcriptional regulation (8). Recently, mutations in various genes encoding different subtypes of the SWI/SNF complex have been identified in $20 \%$ of different human cancers $(9,10)$. INI1, located on chromosome 22q11.2, is one of the SWI/SNF subtypes and the most well-established member of this pathway (11). Complete loss of the INI1 gene product resulting from biallelic inactivation of the gene through genetic and epigenetic mechanisms has been reported in various paediatric and soft-tissue sarcomas. Increasing neoplasms with INI1 deficiency include paediatric malignant rhabdoid tumours, epithelioid sarcoma, myxoid chondrosarcoma, medullary carcinoma of the kidney, myoepithelial neoplasms $(12,13)$ and the recently described gastrointestinal, pancreatic and sinonasal system carcinoma variants $(13,14)$. Some studies on these carcinomas have demonstrated that INI1 loss is important not only in establishing a diagnosis but also in correlating with poor prognosis (14). To the best of our knowledge, no studies have investigated INI1 loss in type 1 and type 2 EC to date.
Cell membrane adhesion molecules are responsible for the binding of cells to each other and the extracellular matrix, thereby playing a key role in carcinogenesis (15). E-cadherin is a calcium-dependent transmembrane glycoprotein responsible for maintaining the adhesion between epithelial cells. In cancer cells, metastasis is closely related to the epithelial-mesenchymal transition characterised by downregulation of E-cadherin $(16,17)$. A decrease in E-cadherin expression or loss of function of this protein is a sign of metastasis and poor prognosis in various cancers $(18,19)$. As in other carcinomas, E-cadherin appears to be an indicator of unfavourable clinicopathological factors and poor survival in EC. A number of studies in the literature have explained the more aggressive behaviour of type 2 tumours by detecting loss of E-cadherin expression in type $2 \mathrm{EC}$ compared with type 1 $(20,21)$. However, the exact role of E-cadherin in type $2 \mathrm{EC}$ and the mechanisms responsible for the downregulation of this protein have not been fully clarified. Loss of E-cadherin expression has been associated with dedifferentiation, deep myometrial invasion and increased metastatic potential in EC due to the altered tumour suppressor role of this protein (22). E-cadherin mutations have been recognised as an indicator of poor prognosis and shorter life expectancy in patients with various neoplasms including gastric, breast and colon cancer (17).

This study aimed to investigate loss of INI 1 and E-cadherin expression in cases of type 1 and 2 EC, aid in the differential diagnosis by demonstrating the difference in this loss between histological types and to evaluate two markers that can shed light on the carcinogenetic mechanisms that may explain the prognostic difference between these types.

\section{METHODS}

A total of 72 patients diagnosed with EC who underwent hysterectomy in 2012-2020 were enrolled in the study. Paraffinembedded blocks were retrieved from the archive and blocks that contained normal endometrial glands as well as tumour tissue were selected. This is a retrospective study of the archive materials of these patients.

\section{Histopathological Evaluation}

The microscope slides (preparations) of all cases were reviewed to confirm the diagnosis. Clinicopathological data of the malignant cases were collected from medical reports and included patients' age, histopathological tumour type, grade and pattern. All specimens were hysterectomy specimens and grading of EC cases was performed only on hysterectomy specimens.

\section{Immunohistochemistry}

Sections of 4-micron thickness were obtained from 72 formalinfixed, paraffin-embedded tissues for immunohistochemical (IHC) analysis and positive-charged microscope slides were used to avoid tissue shedding. The sections were allowed in an incubator at $60^{\circ} \mathrm{C}$ for an hour and deparaffinised with xylene for $15 \mathrm{~min}$. 
The samples were hydrated through descending-grade series of alcohol and washed in distilled water. Samples were then introduced to a BenchMark XT device. INI1 (Cell Marque, RTU, MRQ-27, Philadelphia, PA, USA) and E cadherin (Cell Marque, RTU, EP700Y, Rocklin, CA, USA) antibodies were applied, and staining was performed subsequently. The samples stained in the automated staining device were covered using fluid-based covering material. Results were evaluated with an Olympus CX41 microscope.

\section{Immunohistochemistry Evaluation}

Clearly observed expression loss in tumour cells was accepted as loss of INI1 expression. Normal mucosal glands, stromal fibroblasts, endothelial cells and inflammatory cells were utilised as internal control.

The intensity of membranous and membranocytoplasmic $\mathrm{E}$-cadherin staining in epithelial cells was graded as 0 (negative), 1 (weak), 2 (moderate) and 3 (strong). For E-cadherin, the percentage of positive-stained cells was graded as $0(0 \%), 1$ (1\%-10\%), 2 (11\%-50\%) and 3 (>50\%). The final score for E-cadherin (0-6) was determined by combining the intensity and percentage scores (23). An internal positive control, which consisted of endometrial epithelium and the adjacent normal glandular epithelium, was used for quality control as well as an internal negative control of adjacent normal endometrial stroma and myometrium.

\section{Statistical Analysis}

Patient demographics and data were analysed using the SPSS 24 programme. Variables were expressed as frequency, percentage, mean (arithmetic mean and median), standard deviation (minmax), tables and graphs. Chi-square test was used to compare patient-related variables in both groups. $\mathrm{P}<0.05$ was considered statistically significant.

The study was approved by the Tekirdağ Namık Kemal University Non-interventional Clinical Trials Ethics Committee (approval number: 2020.92.04.16, approval date: 30.04.2020).

\section{RESULTS}

The mean age of the 72 patients was 63.6 (37-83) years. Diagnosis distribution of the patients is presented in Table 1. A total of 63

\begin{tabular}{|l|l|l|l|}
\hline \multicolumn{2}{|l|}{ Table 1. Diagnosis distribution of the patients } \\
\hline Diagnosis & Number of patients & $\%$ \\
\hline $\begin{array}{l}\text { Endometrioid } \\
\text { carcinoma }\end{array}$ & $\begin{array}{l}\text { Grade 2 } \\
\text { Grade 3 }\end{array}$ & 29 & 87.5 \\
\hline Serous carcinoma & 7 & 4 & 9.7 \\
\hline Clear-cell carcinoma & 1 & 1.4 \\
\hline Dedifferentiated carcinoma & 1 & 1.4 \\
\hline
\end{tabular}

patients had type 1 (endometrioid) tumours and 9 had type 2 (nonendometrioid) tumours. Eight cases with endometrioid carcinoma had squamous differentiation, two had secretory differentiation and five had villoglandular pattern.

IHC staining revealed loss of INI1 expression in eight cases. Three of these cases were serous carcinoma, three were endometrioid carcinoma with villoglandular pattern (two cases of grade 1 and one case of grade 2) and two were grade 3 endometrioid carcinoma. The INI1 and E-cadherin staining of the cases are presented in Figure 1.

Loss of INI 1 expression was detected as $3 / 7$ in serous carcinoma cases, $3 / 5$ in endometrioid carcinoma with villoglandular pattern and 2/4 in grade 3 endometrioid carcinoma.

A significant difference was observed in the loss of INI1 expression between low-grade (G1-G2) tumours and high-grade (G3 and non-endometrioid carcinoma) tumours ( $p=0.004)$.

Microscope slides, i.e. preparations of 30 patients, contained tumour tissue as well as atrophic endometrial glands. None of the cases had loss of INI1 and E-cadherin expression in atrophic glands. While atypical endometrial hyperplasia areas as well as carcinoma areas in $\mathrm{IHC}$ slides were observed of eight patients, none of these cases had loss of INI1 and E-cadherin expression.

The loss of expression was observed only in one case of dedifferentiated carcinoma in IHC staining performed for E-cadherin in 72 cases. There was no loss of INI1 expression in this case. In all other cases (endometrioid carcinoma grade 1, 2, 3 , serous carcinoma and clear-cell carcinoma), E-cadherin staining intensity was $3+$, and staining was observed in more than $50 \%$ of the cells, resulting in a final score of 6 .

\section{DISCUSSION}

Aggressive features of high-grade ECs, resistance to chemotherapy, poor prognosis and extremely high rates of recurrence contribute to increased mortality in patients with EC each year. Surgical treatment, together with chemotherapy and radiotherapy, remains in use in high-grade ECs. A clear understanding of the molecular changes in EC subtypes is expected to facilitate accurate diagnosis and prognosis predictions as well as establish targeted therapeutic strategies. In addition to treatments that target somatic hot-point gene mutations, advances in tumour metabolism and immunotherapy further open new doors for patients with high-grade EC. In this regard, emerging molecular studies should help synthesise new therapeutic inhibitors (24).

Bi et al. (25) applied IHC for INI1 and BRG1 in 10 cases of dedifferentiated EC and 16 cases of undifferentiated EC, identifying loss of BRG1 in 12 cases and INI1 loss in 2. The authors found a significant decrease in survival in cases with loss of INI1 and BRG1 expressions (mean, 4.7 and 22.9, $\mathrm{p}=0.033$ ). They showed that BRG1 and INI1 expressions were not associated with age, myometrial invasion, lymph node status and International 


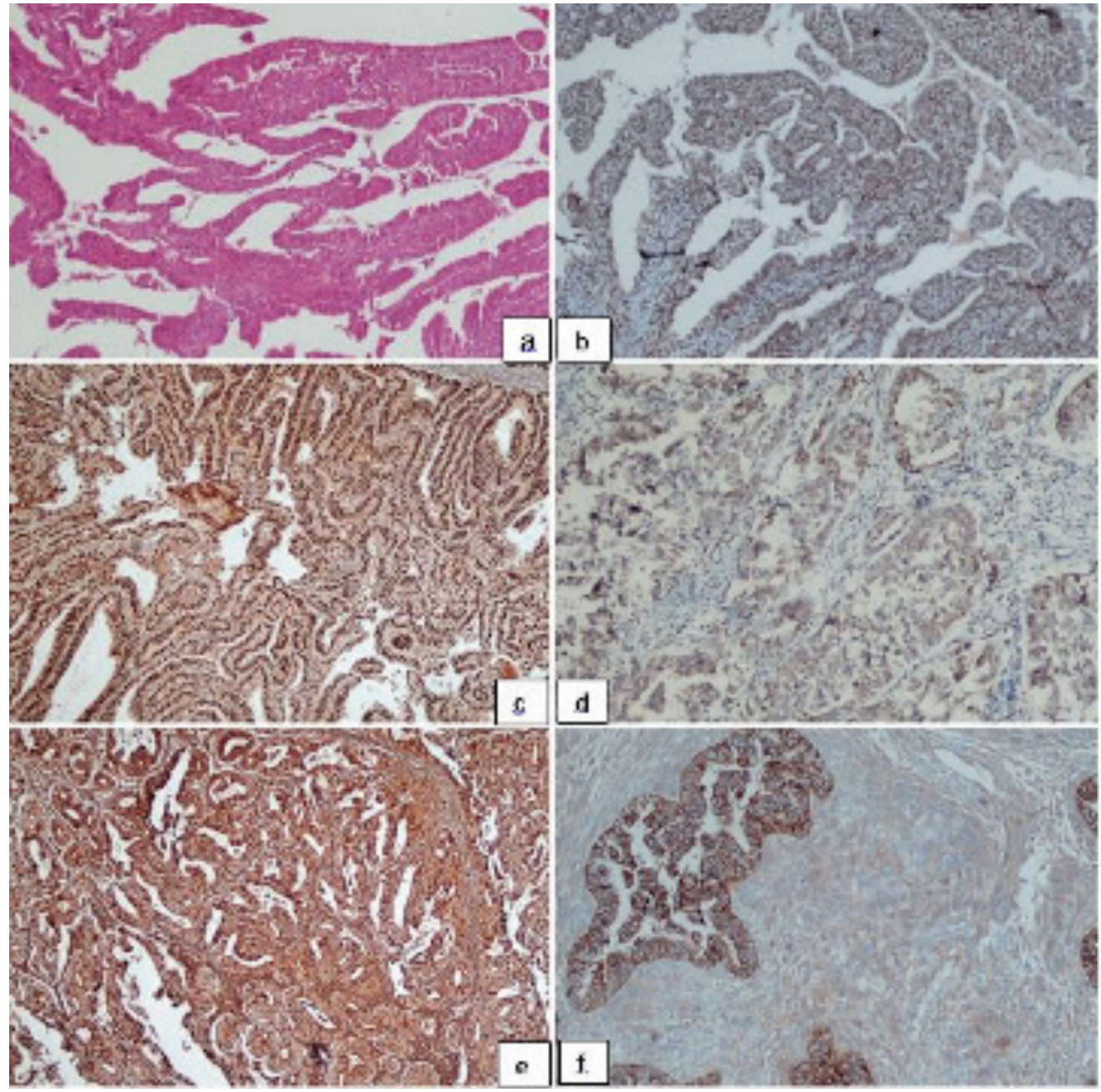

Figure 1. The INI1 and E-cadherin staining of the cases a) endometrioid carcinoma with villoglandular pattern [hematoxylin and eosin staining (H\&E), x100]; b) endometrioid carcinoma with villoglandular pattern, INI1 negative (x100); c) endometrioid carcinoma, INI1 positive (x100); d) serous carcinoma, INI1 negative (x100); e) endometrioid carcinoma, E-cadherin positive (x100); and f) dedifferentiated carcinoma, E-cadherin negative $(x 100)$

Federation of Gynecology and Obsterics (FIGO) stage ( $p=0.437$, $p=0.672, p=0.242, p=0.348$ ). In light of their findings, they recommended routine IHC staining for BRG1 and INI1 in dedifferentiated and undifferentiated ECs to aid the identification of these tumours, distinguishing them from other carcinomas and predicting their clinical prognosis (25).

Strehl et al. (8) applied IHC for INI1 and BRG1 in 24 cases of grade $3 E C$ and two cases of undifferentiated EC, showing diffuse INI1 expression in all cases without any loss of expression. They detected loss of BRG1 expression in only one case, again without any loss in INI1 expression. The authors concluded that SWI/SNF may be effective in the pathogenesis of high-grade ECs (8). Apart from these two studies on high-grade ECs, we have not encountered any other systematic study investigating INI1 loss in ECs.

Loss of INI1 has shown importance in establishing diagnosis as well as in predicting poor prognosis in adult soft-tissue sarcomas, paediatric tumours and newly described carcinomas with INI1 loss (14). In addition to being a prognostic factor, some studies have reported PD-L1 positivity in tumours with loss of INI1 expression $(13,26)$. PD-L1-expressing tumours of different histological types are known to show better response to treatment with immune checkpoint inhibitors than PD-L1-negative tumors (13).

In the present study, a significantly greater loss of INI1 expression was observed in high-grade EC compared with low-grade. This finding confirms that INI1 loss is a poor prognostic factor in this malignancy as in tumours reported in the literature and elucidates on the different pathogeneses seen in high-grade EC. On the contrary, cases of EC with villoglandular pattern are generally assumed to behave like type $1 \mathrm{EC}$. We detected loss of INI1 expression in three out of the five cases of endometrioid carcinoma with villoglandular pattern. We believe that future studies with a greater number of patients may further explore whether the INI1 loss demonstrated in this study is a prognostic 
factor in endometrioid carcinoma with villoglandular pattern and elucidate whether this different morphology stems from the differences in pathogenesis.

In a previous study, patients with atypical endometrial hyperplasia and EC were evaluated for E-cadherin and CD10 by $\mathrm{IHC}$ and correlation between loss of $\mathrm{E}$-cadherin expression and tumour grade as well as FIGO stage was found significant despite patients' age, specimen type, tumour pattern or histopathological tumour types (22). Another study investigated type 1 and 2 ECs for $\mathrm{E}$-cadherin and $\mathrm{N}$-cadherin and the loss of E-cadherin expression was statistically significant among type 2 ECs rather than type $1 \mathrm{ECs}(p=0.007)$. Aggressive behaviour was found to be related to loss of E-cadherin in type $2 \mathrm{ECs}$, but other clinicopathological features were lacking. In addition, decreasing E-cadherin expression in lymphovascular tumour cells were shown be another finding of this study. Myometrial invasion in ECs was suggested to be caused by the epithelial-mesenchymal transition mechanism (21).

Vroobel and Attygalle (27) applied IHC for E-cadherin, EMA and DNA mismatch repair proteins in three cases of undifferentiated and dedifferentiated EC, showing loss of E-cadherin expression in dedifferentiated EC cases and suggested that this could be utilised in routine practice to aid in the differential diagnosis. Consistent with the literature, loss of E-cadherin expression was identified in a dedifferentiated EC case in the study presented herein. We believe this marker may have diagnostic value in dedifferentiated EC. However, further studies with a larger number of patients are required to confirm this notion.

We did not observe loss of E-cadherin expression across EC cases evaluated in this study. Studies have shown that E-cadherin levels are associated with aggressive behaviour, poor survival and increased metastasis $(20,21)$. This may result from the fact that studies in the literature often rely on mRNA levels of E-cadherin and may be due to the differences at clone level in studies utilising $\mathrm{IHC}$. Our findings in this aspect may be associated with the clone we used in the study, which is deemed not appropriate for ECs. We believe this study may be repeated using the clones described in the literature and more robust results may be achieved in the presence of further parameters such as FIGO staging, myometrial invasion rate, survival and metastasis.

\section{Study Limitations}

The low number of patients with type $2 \mathrm{EC}$ is one of the limitations of our study. More meaningful results could be obtained with highgrade ECs. Our study only compares the difference in staining of markers between histopathological types. We did not evaluate the relation of INI-1 and E-cadherin loss with prognostic parameters such as myometrial invasion and lymph node metastasis. More significant results can be obtained by comparing the staining of markers with prognostic parameters and by survival analysis.

\section{CONCLUSION}

Our results confirm that the SWI/SNF chromatin remodelling complex plays a role in the pathogenesis of high-grade EC and type $1 \mathrm{EC}$ with villoglandular pattern. A few studies have investigated this subject in the literature. We believe studies on INI1 expression in EC cases, together with survival analysis, are warranted in these patients. Furthermore, our study should guide future studies in investigating PD-L1 expression in cases of EC with villoglandular pattern and high-grade ECs, where we identified loss of INI1 expression. This may allow encouraging outcomes with immune checkpoint inhibitors added to treatment of these tumours, especially in high-grade ECs with poor survival.

Ethics Committee Approval: The study was approved by the Tekirdağ Namık Kemal University Non-interventional Clinical Trials Ethics Committee (approval number: 2020.92.04.16, approval date: 30.04.2020).

Informed Consent: Retrospective study.

Peer-review: Externally peer-reviewed.

Financial Disclosure: The author declared that this study has received no financial support.

\section{REFERENCES}

1. Piulats JM, Guerra E, Gil-Martín M, Roman-Canal B, Gatius S, SanzPamplona R. Molecular approaches for classifying endometrial carcinoma. Gynecol Oncol 2017; 145: 200-7.

2. Yeramian A, Moreno-Bueno G, Dolcet X, Catasus L, Abal M, Colas E, et al. Endometrial carcinoma: molecular alterations involved in tumor development and progression, Oncogene 2013; 32: 403-13.

3. Kurman RJ, Carcangiu ML, Herrington CS, Young RH. WHO classification of tumors of the female reproductive organs. WHO classification of tumors. Lyon: IARC Press; 2014.

4. Hamilton CA, Cheung MK, Osann L, Chen L, Teng NN, Longacre TA, et al. Uterine papillary serous and clear cell carcinomas predict for poorer survival compared to grade 3 endometrioid corpus cancers. Br J Cancer 2006; 94: 642-6.

5. Matias-Guiu X, Prat J. Molecular pathology of endometrial carcinoma, Histopathology 2013; 62: 111-23.

6. Esteller M, Garcia A, Martinez-Palones J, Xercavins J, Reventos J. Clinicopathologic features and genetic alterations in endometrioid carcinoma off the uterus with villoglandular differentiation. Am J Clin Pathol 1999; 111: 336-42.

7. Ambros RA, Ballouk F, Malfetano JH, Ross JS. Significance of papillary (villoglandular) differentiation in endometrioid carcinoma of the uterus. Am Surg Pathol 1994; 18: 557-69.

8. Strehl JD, Wachter DL, Fiedler J, Heimerl E, Beckmann MW, Hartmann A, et al. Pattern of SMARCB1 (INI1) and SMARCA4 (BRG1) in poorly differentiated endometrioid adenocarcinoma of the uterus: analysis of a series with emphasis on a novel SMARCA4-deficient dedifferentiated rhabdoid variant. Ann Diagn Pathol 2015; 19: 198-202.

9. Wang X, Haswell JR, Roberts CW. Molecular pathways: SWI/SNF (BAF) complexes are frequently mutated in cancer-mechanisms and potential therapeutic insights. Clin Cancer Res 2014; 20: 21-7.

10. Shain $A H$, Pollack JR. The spectrum of SWI/SNF mutations, ubiquitous in human cancers. PLoS One 2013; 81: e55119.

11. Judkins AR. Immunohistochemistry of INI1 expression: a new tool for old challenges in CNS and soft tissue pathology. Adv Anat Pathol 2007; 14: 335-9.

12. Hollmann TJ, Hornick JL. INI1-deficient tumors: diagnostic features and molecular genetics. Am J Surg Pathol 2011; 35: 47-63.

13. Forrest SJ, Al-Ibraheemi A, Doan D, Ward A, Clinton CM, Putra J, et al. Genomic and immunologic characterization of INI1-deficient pediatric cancers. Clin Cancer Res 2020; 26: 2882-90. 
14. Tang SJ, Zhai CW, Yuan CC, Zhang JH, Wang SY. SMARCB1 (INI1)deficient sinonasal carcinoma: a clinicopathological analysis of six cases. Zhonghua Bing Li Xue Za Zhi 2020; 49: 47-51.

15. Florescu MM, Pirıcı D, Sımıonescu $C E$, Stepan $A E$, Mărgărıtescu $C$, Tudorache $S$, et al. E-cadherin and $\beta$-catenin immunoexpression in endometrioid endometrial carcinoma. Rom J Morphol Embryol 2016; 571: $1235-40$.

16. Xiong S, Klausen C, Cheng JC, Leung PCK. Activin B promotes endometrial cancer cell migration by downregulating $\mathrm{E}$-cadherin via SMAD-independent MEK-ERK1/2-SNAIL signaling. Oncotarget, 2016; 28: 40060-72.

17. Wong SHM, Fang CM, Chuah LH, Leong CO, Ngai SC. E-cadherin: Its dysregulation in carcinogenesis and clinical implications. Crit Rev Oncol Hematol 2018; 121: 11-22.

18. Costa LC, Leite CF, Cardoso SV, Loyola AM, Faria PR, Souza PE, et al. Expression of epithelial-mesenchymal transition markers at the invasive front of oral squamous cell carcinoma. J Appl Oral Sci 2015; 23: 169-78.

19. Choi JE, Bae JS, Kang MJ, Chung MJ, Jang KY, Park HS, et al. Expression of epithelial-mesenchymal transition and cancer stem cell markers in colorectal adenocarcinoma: clinicopathological significance. Oncol Rep 2017; 38: 1695-705.

20. Setiawan VW, Yang HP, Pike MC, McCann SE, Yu H, Xiang YB, et al. Type I and II endometrial cancers: have they different risk factors? J Clin Oncol 2013; 31: 2607-18.

21. Rubeša-Mihaljevic R, Babarovic E, Vrdoljak-Mozetic D, StembergerPapic S, Klaric M, Krasevic M, et al. The immunohistochemical pattern of epithelial-mesenchymal transition markers in endometrial carcinoma. Appl Immunohistochem Mol Morphol 2019; 53: 164-72.

22. Youssef MY, Mohamed MA. Could e-cadherin and cd10 expression be used to differentiate between atypical endometrial hyperplasia and endometrial carcinoma? Appl Immunohistochem Mol Morphol 2019; 38: 128-37.

23. Jie $D$, Zhongmin $Z$, Guoging $L$, Sheng $L$, Yi Z, Jing W, et al. Positive expression of LSD1 and negative expression of E-cadherin correlate with metastasis and poor prognosis of colon cancer. Dig Dis Sci 2013; 58: 1581-9.

24. Zhang L, Kwan SY, Wong KK, Soliman PT, Lu KH, Mok SC. pathogenesis and clinical management of uterine serous carcinoma. Cancers(Basel) 2020; 12: 686.

25. Bi R, Yu L, Tu XY, Ge HJ, Cheng YF, Chang B, et al. Expression of SMARCA4(BRG1) and SMARCB1(INI1) in dedifferentiated and undifferentiated endometrial carcinomas and their correlations with clinicopathological features]. Zhonghua Bing Li Xue Za Zhi 2019; 8: 590-5.

26. Abro B, Kaushal M, Chen L, Wu R, Dehner LP, Pfeifer JD, et al. Tumor mutation burden, DNA mismatch repair status and checkpoint immunotherapy markers in primary and relapsed malignant rhabdoid tumors. Pathol Res Pract 2019; $215: 152395$.

27. Vroobel KM, Attygalle AD. Sarcomatous Transformation in Undifferentiated/ Dedifferentiated Endometrial Carcinoma: An Underrecognized Phenomenon and Diagnostic Pitfall. Int J Gynecol Pathol 2020; 39: 485-92. 